\title{
Leishmaniasis in Sri Lanka: a decade old story
}

\author{
HVYD Siriwardana, ${ }^{1}$ PH Chandrawansa, ${ }^{2}$ G Sirimanna, ${ }^{3}$ ND Karunaweera ${ }^{1}$ \\ Sri Lanka Journal of Infectious Diseases 2012 Vol.2(2):2-12 \\ DOI: DOI: http://dx.doi.org/10.4038/sljid.v2i2.4420
}

Key words: Leishmaniasis, Sri Lanka, skin lesions, L.donovani

\begin{abstract}
Visceral leishmaniasis is a vector-borne protozoan disease targeted for elimination from the Indian subcontinent by year 2020. Sri Lanka is a new focus of human leishmaniasis caused by a genetically distinct variant of Leishmania donovani, which is the species more widely known to cause visceral leishmaniasis (VL). The clinical entity that is most frequent in Sri Lanka is cutaneous leishmaniasis (CL), though a few cases of muco-cutaneous (MCL) and VL have also been reported in the recent past. In CL, papules, nodules, ulcerating nodules and ulcers occur mainly as single lesions on exposed body areas of affected individuals. A wide age range is affected including both sexes. The potential for visceralization in the cutaneous variant of $L$. donovani in Sri Lanka is not known. There is no evidence for a serological response in CL patients who have demonstrated negative for rK 39 antibodies in sera, (rk39 test being the recommended test for VL detection), while MCL and VL cases have been rK39 positive.
\end{abstract}

Phlebotomus argentipes, the vector of $L$. donovani in other parts of the world is a widely prevalent insect in almost all parts of Sri Lanka. Studies are underway for vector identification. L.donovani is transmitted between this vector and the human host, the only known host for this species. However, domestic dogs have shown the presence of Leishmania parasites and also the presence of anti - L. donovani antibodies in their sera, providing primary evidence for the likely presence of an animal reservoir in Sri Lanka. Field-based risk factor studies have shown that there is peri-domestic as well as zoonotic/outdoor transmission cycles in different parts of the country.

At present patients are detected mainly passively based on self referrals. Only a proportion of these patients proceed for pre-treatment laboratory confirmation due to the lack of freely available investigation facilities. Leishmaniasis was made a notifiable disease in Sri Lanka in 2008. Action plan for its control was drawn up with primary involvement of the Ministry of Health and other stake holders in 2008. Preventive and control activities are required to be put in place sooner rather than later. Enhanced case detection and active treatment are of prime value in controlling L.donovani infections. Availability of cost effective and field friendly diagnostic services in a decentralized manner, timely case management and vector control using appropriate protocols are necessary. To achieve this, a considerable amount of information is already available, and further research is needed to fill in the essential gaps.

\footnotetext{
${ }^{1}$ Department of Parasitology, Faculty of Medicine, University of Colombo, Sri Lanka

${ }^{2}$ National Hospital of Sri Lanka

Address for correspondence: Department of Parasitology, Faculty of Medicine, University of Colombo, Sri Lanka Phone; 0094112 695300, FAX: 0094112699284 email : yayureka@hotmail.com
} 


\section{The disease leishmaniasis}

Human leishmaniasis is a vector-borne protozoan infection that clinically manifests in 3 main forms i.e. cutaneous (CL), muco-cutaneous (MCL) and visceral leishmaniasis (VL), mainly due to the different Leishmania species with affinity to varying organs of the host. The disease has resulted in a huge global burden and is listed as one of the eight major neglected tropical parasitic diseases (1). The World Health Organization (WHO) has targeted elimination of its most virulent form, VL, from the Indian subcontinent by year $2020 .{ }^{1}$ In spite of these attempts, the number of leishmaniasis endemic sites is ever expanding with new foci and new epidemics in endemic sites being continuously reported at a global scale. ${ }^{2,3,4}$ New genetic variants of Leishmania sp. are also being continuously reported. ${ }^{5,6}$ Sri Lanka is the newest reported focus of leishmaniasis in the Indian subcontinent. ${ }^{7}$ The disease is caused by the most virulent visceralizing species, L.donovani. ${ }^{8}$

\section{Growing problem of leishmaniasis in Sri Lanka}

In the 1990's, leishmaniasis in Sri Lanka took the nature of an imported disease where cases were reported mainly among overseas employees returning to the country. ${ }^{9}$ Local transmission of leishmaniasis was first evident from southern Sri Lanka in $1992 .{ }^{10}$ Few sporadic cases were detected locally until 2001, ${ }^{11}$ when the onset of the on-going outbreak of leishmaniasis in Sri Lanka became evident. ${ }^{7}$ Since then, over 2500 clinically suspected cases have been referred to the University of Colombo (UOC) alone for disease confirmation (DPC central data base). The disease has been made notifiable since 2008 and a national action plan has been developed for leishmaniasis control. ${ }^{12}$ However, much work still remains to be done.

\section{What is known so far?}

\section{The patient and the parasite}

Clinical features of the initial patients in this epidemic were reported in 2003 confirming the presence of locally acquired CL in Sri Lanka. ${ }^{7}$ Since then, cases of CL are being continuously reported to the health care institutions of this country. In the initial phase of the outbreak, more males in the 21-40 year age group were affected and a clear majority of them were soldiers working in Northern Sri Lanka. ${ }^{7}$ Lesions were mainly single and occurred on the exposed body areas in the form of either papules, nodules, ulcerating nodules and complete ulcers that were seen in almost equal proportions. ${ }^{7}$ Since then, results of clinical characterization studies, which described the cases presenting during the first half of the epidemic have been made available at different times during the decade. ${ }^{7,13,14,15,16}$ These studies highlight a widening of case distribution in the country to include more and more districts, though in general, other clinical and demographical features remained nearly the same as reported earlier.

With the continued increase in the reported CL case numbers, biochemical studies were started in 2001 to identify the local Leishmania parasite species and were completed 2 years later confirming the presence of L.donovani MON 37 in Sri Lanka. ${ }^{17}$ L. donovani is usually the causative organism of VL. Therefore, parasite species was revisited, using molecular biological methods that investigated the parasite genome. These studies confirmed the finding of L.donovani in $2007 .{ }^{8}$ Furthermore, parasite studies have shown that the local L. donovani is genetically different from members of the same species complex found in previously identified 
endemic settings. ${ }^{8}$ However, the scientific reasons for the mismatch of a visceralizing parasite with a clinical picture of $C L^{16}$ still remain obscure.

Clinical manifestations of CL however, are diverse, ranging from acne-form papules to complete ulcers. A range of atypical manifestations have also been reported later on in some studies. $^{16,18}$ Increasing the complexity of this picture, MCL was first reported in $2005,{ }^{19}$ followed by the first report of VL two years later. ${ }^{20}$ Soon after, a second case of MCL with lip, buccal mucosal and nasal septum lesions was reported from Northern Sri Lanka. ${ }^{21}$ VL patients presented with asymptomatic hepato-splenomegaly and parasites were isolated from the bone marrow. Immune response patterns of few local patients were studied in 2010 using rk 39 assays. Twenty four (24) cases of CL studied were rk 39 negative while MCL and VL cases were positive. ${ }^{16} \mathrm{rK} 39$ is a $L$. donovani specific antibody and the assay is the recommended serological test for diagnosis of VL.

\section{The vector and animal reservoirs}

L. donovani is transmitted by its natural vector Phlebotomus argentipes, which is generally known as the sand fly ("Hohaputuwa" in the local language - Sinhala). Sand fly research in Sri Lanka has a long history and the presence of sand flies has been reported since as early as the 1940 's. ${ }^{22,23,24}$ More recent information on Phlebotomine sand flies in Sri Lanka is available since $2005^{25}$ where small numbers of Sergentomyia ceylanica were also identified. ${ }^{26}$ Disregarding the negligible percentages of this species, $P$. argentipes could be the putative local vector of leishmaniasis in this country. Confirming this, reports last year revealed that all 3 members of the Phlebotomus complex, i.e. Phlebotomus glaucus, Ph. argentipes sensu stricto and Ph. annandalei are prevalent in Northern Sri Lanka. ${ }^{27}$ Sand fly behaviour has also been studied in the 1970's, 1990's and in 2005..$^{23,24,25,26}$ Both zoophilic and anthropophilic behaviour patterns have been identified in these studies.

Humans were the only known reservoir for L.donovani. However, recently (2009) Leishmania parasites were detected by PCR in dogs from the central highlands, providing preliminary evidence for possible animal involvement in Sri Lanka. ${ }^{28}$ Following this, the serological studies carried out in southern Sri Lanka provided evidence for the presence of anti L.donovani rK 39 antibodies in domestic dogs which supports their reservoir host status ${ }^{29}$ (authors' unpublished observations).

\section{Risk factors and transmission cycle}

Factors affecting Leishmania transmission have been studied at different sites in Sri Lanka. Population-based case control studies were started in 2002-3 which determined prevalence and risk factors of leishmaniasis. ${ }^{16,30}$ Baseline evidence for zoonotic transmission in the North and peri-domestic transmission in coastal plain in the south, at least in the study sites, was obtained in these studies. Young adults between 20-40 years, male sex and spending more time in outdoor activities were associated with leishmaniasis in northern Sri Lanka, favouring an outdoor transmission. ${ }^{30}$ In contrast, the studies carried out more recently (2006) in southern Sri Lanka showed age between 11-40 years, household clustering and housing conditions were associated with increased risk of acquiring leishmaniasis, favouring a peri-domestic transmission. ${ }^{16}$ 


\section{Case detection and management}

Apart from a few active case detection programmes carried out to determine risk factors and prevalence, the main mode of case detection remains passive, as self referral of affected individuals to a health care setting. Laboratory investigations necessary for Leishmania diagnosis have been established during the last decade. Light microscopy, ${ }^{31}$ parasite in-vitro isolation, field friendly culture techniques ${ }^{17,32,33,34}$ and PCR methods ${ }^{34}$ for leishmaniasis diagnosis have been established in a few laboratories. The rK 39 assay is also used in some laboratories for diagnosis of VL, though availability is limited. ${ }^{16}$

\section{Could this situation be taken lightly and ignored?}

Although L.donovani is generally known to cause VL, occasional CL, ${ }^{35,36} \mathrm{MCL}^{37}$ and CL with concurrent VL have also been reported. ${ }^{36}$ Furthermore, an epidemic similar to the Sri Lankan epidemic with CL caused by L.donovani was reported in northern India in $2005 .^{5}$ This might indicate the likelihood of more stable cutaneous variants of L.donovani getting established at different endemic settings.

The dangerous possibility of visceralization of the Sri Lankan cutaneous variant cannot be disregarded until the extent of the problem is scientifically and adequately measured over a reasonable period of time. Recent reports of VL and MCL in the country already points towards this possibility. On the other hand, multiple genetic variants of L.donovani may be responsible for different clinical forms in Sri Lanka where initial CL cases remain confined to skin without visceralization.

Usually primary infections result in immunity and resistance to re-infection by homologous Leishmania species. ${ }^{38} \mathrm{CL}$ patients may completely heal spontaneously or with treatment. Achieving a parasitological cure is difficult in leishmaniasis and remaining parasites may stay dormant. ${ }^{39} \mathrm{CL}$ is also known to recur in about $1 \%$ of patients. ${ }^{40} \mathrm{CL}$ has already recurred in a few patients in the local setting (DPC data base). Cutaneous leishmaniasis caused by $L$. donovani is also known to recur in other parts of the world. ${ }^{41}$

It is not known whether initial cutaneous infections will remain confined to the skin in the Sri Lankan population. Emergence of MCL cases and VL cases in the island may indicate the ability of local Leishmania parasites to visceralize, metastasize to mucosa or to the existence of multiple variants. Increase in parasite virulence and the non-immune status of the indigenous population with increased vulnerability may help establishment of MCL and VL causing variants. Historical information already provides supportive evidence for the presence of visceralizing parasite variants in the country since long. ${ }^{42}$ The number of VL cases may be grossly under diagnosed in Sri Lanka, due to low transmission levels of VL, asymptomatic nature $^{43}$ and lack of clinical suspicion.

An established though obscure Leishmania transmission cycle/s may have existed in this country from early years. ${ }^{42}$ Less severe and mostly asymptomatic nature of human infections, low rates of transmission and regular anti-malarial spraying activities may have kept the disease at an unnoticeable level until it flared up in early 2000. This apparent increase in the number of cases may have been due to changes in human behaviour related to the civil war during that time, which made soldiers move into previously uninhabited jungle areas, which in turn may have exposed them to sylvatic cycles. Cessation of insecticide spraying for malaria control, populations coming into close contact with scrub jungle based zoonotic cycles, 
recently introduced developmental projects, military activities and the influx of non-immune people to this existing transmission cycles may have contributed in maintaining and increasing the number of cases.

The presence of two main foci of Leishmania transmission within the island can be proposed, one in the north and other in the south. The presence of zoophilic Phlebotomine species and the outdoor associated risk factors identified in the north point towards a possible existence of a zoonotic transmission cycles, at least in parts of Sri Lanka, while transmission may take place peri-domestically in southern Sri Lanka. Differences in transmission characteristics and specially the presence of a zoonotic cycle make it difficult to prescribe a homogenous control programme for the island. It may be necessary to define these characteristics in detail and appropriate methods should be defined on a regional basis. A single programme introducing the same activities in different regions may be inappropriate.

\section{Towards disease control, gaps in knowledge and future needs}

Different management and control strategies have been reviewed at times in the world. ${ }^{44}$ Early case detection and treatment, vector control (managing the sand flies that transmit the parasite) and communication and education within endemic communities are the three main strategies highlighted by WHO for elimination of VL. ${ }^{1}$ WHO has set a target for $100 \%$ detection and treatment of VL and its complete elimination in the Indian subcontinent by year $2020 .{ }^{1}$

It is a timely need to identify and adopt correct preventive and control strategies for Sri Lanka sooner rather than later, before more virulent strains are established. These strategies need to be developed in an evidence-based manner. ${ }^{12}$

\section{Enhanced clinical suspicion}

Accurate and complete case detection and treatment that helps to reduce the parasite reservoir are invaluable. Raising awareness among the general public to enhance self referrals, a detailed understanding of clinical characteristics among clinicians and primary health care workers, surveillance for changing clinical patterns at tertiary level, active case detection programmes to improve case detection and availability and decentralization of clinical expertise, knowledge and facilities will ensure improved case detection. Clinical suspicion of leishmaniasis in a patient should be considered early rather than by exclusion of other illnesses. With the emergence of MCL, VL and diversity in CL manifestations, the disease should be considered in the differential diagnosis not only in the dermatological and general medical settings, but in the dental and ENT surgical settings as well.

\section{Need for laboratory confirmation?}

Case detection by health authorities in Sri Lanka is mainly dependant on self referrals made by patients themselves to the health care institutions, apart from the few screening programmes carried out in an ad hoc manner. ${ }^{18}$ Diagnosis is mainly established on clinical grounds, though its reliability in diagnosing atypical skin manifestations, chronic lesions and resistant cases is doubtful and debatable. Only a proportion of cases proceed for laboratory-based confirmation prior to treatment. Unavailability of laboratory facilities and technical expertise within easy reach is the key limiting factor faced by clinicians. Light microscopy remains the mainstay for diagnosis in spite of the limited availability of technical expertise ${ }^{31}$. Parasite in-vitro isolation ${ }^{32,33}$ and PCR is also limited to few laboratories ${ }^{34}$ in the island. 
However, parasitological diagnosis is advisable prior to treatment as there are no pathognomonic clinical features in leishmaniasis, ${ }^{44}$ clinical patterns are diverse ${ }^{40}$ and late lesions or treated lesions have a greater chance of producing false negative results, complicating the decision making procedure for clinicians. Investing in development of inexpensive and simple not so technically demanding laboratory procedures and making them available to remote areas in the country will be useful. Serological assays can also be particularly useful in the context of emerging MCL and VL in the island.

\section{Treat or leave to self-heal?}

Sodium stibogluconate - a drug now known to have over a $60 \%$ resistance rate ${ }^{48}$ and cryotherapy, a treatment modality used for many other dermatological conditions are used to treat leishmaniasis in Sri Lanka. Sodium stibogluconate is available for leishmaniasis treatment only in some dermatology clinics in Sri Lanka.

Treatment outcome of a disease caused by a genetically distinct and understudied parasite variant in Sri Lanka can be different from those that have been recorded from other parts of the world. Unnecessary administration of costly and toxic antileishmanials that require hospitalization and close monitoring should be avoided. Infections with $L$. donovani require treatment with specific anti-leishmanials ${ }^{45}$ while self- limiting forms of CL could be left to self cure. ${ }^{40}$ Response of dermotropic $L$. donovani to standard antileishmanial therapy has shown great variability. Clinical improvement is possible ${ }^{5,38,46}$ while recurrences after several months and after treatment of first recurrence ${ }^{41}$ have also been reported. Therefore, managing a case of CL caused by L. donovani is not straight forward and should be undertaken with utmost caution. Self cure can promote natural immunity against future infections with Leishmania species and treatment may down-regulate these mechanisms. Inappropriate use of drugs, and substandard formulations can result in drug resistance. ${ }^{47}$ India already reports over $60 \%$ drug resistant rate for pentavalent antimonials. ${ }^{48}$ In addition, such irrational drug usage can prevent development of host immunity resulting in visceralization or it can also trigger local spread of a lesion. ${ }^{49}$

The pros and cons of drug treatment of cutaneous $L$. donovani in Sri Lanka remain debatable. Meanwhile, VL and MCL forms definitely require antileishmanial therapy. Carefully designed clinical trials and patient follow-up studies are necessary to decide how the different clinical forms of leishmaniasis in Sri Lanka progress with time. CL may self heal in time, disseminate, recur or develop drug resistance. Delay in cure could also leave a wider window for infection transmission. Therefore, until such evidence is available to the country, treatment of CL in Sri Lanka is advisable due to the dangerous nature of the causative species, to prevent secondary lesions, disfigurement and extension and reduce the risk of spread. The question arises with regard to the appropriate drug/s and protocol selection. To answer such questions multi centre clinical trials on affordable drugs are required. Limited and irregular availability of specific antileishmanials in the curative sector is a major drawback in this regard.

There is a varying sensitivity of usually dermotropic Leishmania species to currently available antimonials and/or combination therapy. ${ }^{50}$ Paucity of properly designed and satisfactorily conducted clinical trials can result in lack of evidence for potentially beneficial treatments. Sodium stibogluconate and meglumine antimoniate are the two main widely used first line antimony compounds since the 1920s. In the Old World, the usefulness of first line drugs for L.donovani are becoming more and more limited. Combination therapy on the other hand helps 
to prevent drug resistance, reduce toxic side effects, reduce the required doses of each drug and increase the spectrum of activity. ${ }^{51,52}$ Therefore, combination treatment is an option that warrants careful consideration for clinical trials in Sri Lanka. Miltefosine is a newer oral antileishmanial which is being increasingly used for the treatment of L.donovani infections in other parts of the world. However the suitability of these options in the local setting needs to be explored with utmost care. Resistance to currently available drugs is not yet reported in Sri Lanka. Once the treatment protocols are defined, strict adherence, timely initiation, completion of the full regime and post treatment case follow up need to be practised. Incomplete treatment of initial disease can enhance recurrence, resistance or visceralization of CL and MCL.

\section{Vector management and transmission control}

Primary preventive measures such as proper clothing, use of insect repellents and avoiding outdoor activities during generally known peak biting hours would be of primary importance to prevent sand fly bites until specific activities are defined. Improvement of housing conditions would also reduce the risk of acquiring infection.

Secondly, insecticides would be necessary to control sand fly populations. However, such efforts should be initiated with strong scientific back up and need to be continued for adequate time before cessation. Unnecessary introduction of insecticides and premature discontinuation can result in more severe epidemics. Our neighbouring country India provides not so pleasant historical evidence regarding the reappearance of the disease probably in greater proportions as a result of such activities. ${ }^{53}$ A similar picture was observed with regard to malaria in Sri Lanka where vigorous and frequent spraying of DDT brought about near eradication of malaria in the 1960 's due to a reduction in vector populations before the case numbers increased in subsequent years.

Studies reported in 2005 indicate that there is a potential for the local sand fly species to develop insecticide resistance. ${ }^{25}$ Tolerance to DDT in $P$. argentipes has been reported from several endemic areas in India. ${ }^{54}$ Treatment of hosts (humans and the dogs) is recommended for zoonotic L. donovani/ infantum infections in the Old World. Long lasting impregnated bed nets (LLIN), indoor residual spraying (IRS) are proven useful for the control of sand flies in the Indian sub-continent. ${ }^{55}$ Integrated vector management that combines multiple methods suited for a particular disease focus has become the method of choice during the recent past. ${ }^{44}$ Studies on local vector incrimination, host preferences, biting, resting and breeding patterns and insecticide susceptibility are needed to fill essential gaps in knowledge. An effective control programme also requires information on regional disease transmission characteristics, socio-demographic and clinical characteristics of the affected populations with disease prevalence and risk factors. Active case detection programmes carried out to cover larger areas in affected provinces would be of importance. Recent report of locally acquired visceral leishmaniasis in Sri Lanka further highlights the importance of such activities. ${ }^{56}$

\section{Conclusion}

A multi-disciplinary approach including improved case detection at community level, strict adherence to case management protocols and control activities, continued surveillance and regular progress evaluation are necessary for effective management and control of leishmaniasis in Sri Lanka. In achieving these goals, the attitudes and compliance of the service recipient community should not be disregarded. Behavioural Change Communication 
where education of people to accept and participate in control programmes would be useful in this regard.

\section{References}

1. Accelerated work to overcome the impact of neglected tropical diseases. A roadmap for implementation. WHO (2012). Geneva, Switzerland. Accessed on line at http://whqlibdoc.who.int/hq/2012/WHO_HTM_NTD_2012.1_eng.pdf (2012.04.20)

2. Rhajaoui M, Fellah H, Pratlong F, Dedet JP and Lyagoubi M. Leishmaniasis due to Leishmania tropica MON-102 in a new Moroccan focus. Trans of Royal Soc of Trop Med and Hyg 2004; 98(5):299-301. doi:10.1016/S0035-9203(03)00071-3

3. Sharifi I, Poursmaelian S, Aflatoonian MR, Ardakani RF, Mirzaei M and Fekri AR et al. Emergence of a new focus of anthroponotic cutaneous leishmaniasis due to Leishmania tropica in rural communities of Bam district after the earthquake, Iran. Trop Med Int Health 2011; 16(4):510-513. doi:10.1111/j.1365-3156.2011.02729.x

4. Giorgobiani E, Chitadze N, Chanturya G, Grdzelidze M, Jochim RC and Machablishvili A. et al. Epidemiologic aspects of an emerging focus of visceral leishmaniasis in Tbilisi, Georgia. PLoS Negl Trop Dis 2011 Dec; 5(12): e1415. doi:10.1371/journal.pntd.0001415

5. Sharma N, Mahajan VK, Kanga A, Sood A, Katoch VM, Mauricio I et al. Localized cutaneous leishmaniasis due to Leishmania donovani and Leishmania tropica: preliminary findings of the study of 161 new cases from a new endemic focus in Himachal Pradesh, India. Am J of Trop Med and Hyg 2005; 72(6):819-824. No doi

6. Gouzelou E, Haralambous C, Amro A, Mentis A, Pratlong F and Dedet JP. et al. Multilocus microsatellite typing (MLMT) of strains from Turkey and Cyprus reveals a novel monophyletic L. donovani sensu lato group. PLoS Negl Trop Dis 2012 Feb; 6(2):e1507. doi:10.1371/journal.pntd.0001507

7. Siriwardana HVYD, Udagedara C and Karunaweera ND. Study on clinical patterns, risk factors and efficacy of cryotherapy in cutaneous leishmaniasis in Sri Lanka. Cey Med J 2003; 48 (1):10-12. doi: http://dx.doi.org/10.4038/cmj.v48i1.3386

8. Siriwardana HV, Noyes HA, Beeching NJ, Chance ML, Karunaweera ND and Bates PA. Leishmania donovani and cutaneous leishmaniasis, Sri Lanka. Emerg Infect Dis 2007; 13(3):476-8. doi:10.3201/eid1303.060242

9. Naotunne $\mathrm{T}$ de $\mathrm{S}$, Rajakulendran $\mathrm{S}$ and Abeywichrema $\mathrm{W}$ et al. Cutaneous leishmaniasis in Sri Lanka. An imported disease linked to the Middle East and African employment boom. Trop Geogr Med 1990; 42:72-74. No doi

10. Athukorale DN, Seneviratne JKK, Ihalamulla RL and Premaratne UN. Locally acquired cutaneous leishmaniasis in Sri Lanka. J Trop Med 1992; 95:432-433. Senevirathne JKK, Siriwardena S, Rathnathunge N and Wijesundera Mde S. Locally acquired leishmaniasis from Central Sri Lanka: a case report. Kandy Medical Journal 1995; 4(2):54-56. No doi

11. Proceedings of International Colloquium on Leishmaniasis in Sri Lanka. In: Abstracts and National Action Plan., 2009.

12. Nawaratna SS, Weilgama DJ, Wijekoon CJ, Dissanayake M and Rajapaksha K. Cutaneous leishmaniasis, Sri Lanka. Emerg Infect Dis 2007; 13(7):1068-1070. doi:10.3201/eid1307.060773

13. Rajapaksa US, Ihalamulla RL, Udagedera C and Karunaweera ND. Cutaneous leishmaniasis in Southern Sri Lanka. Trans R Soc Trop Med Hyg. 2007;101(8):799-803 
14. Samaranayake TN, Dissanayake VH and Fernando SD. Clinical manifestations of cutaneous leishmaniasis in Sri Lanka - possible evidence for genetic susceptibility among the Sinhalese. Ann Trop Med Parasitol 2008; 102(5):383-390. doi:10.1179/136485908X300779

15. Siriwardana HVYD, Thalagala N and Karunaweera ND. Clinical and epidemiological studies on the cutaneous leishmaniasis caused by Leishmania (Leishmania) donovani in Sri Lanka. Ann Trop Med Parasitol 2010 104(3):213-223 doi:10.1179/136485910X12647085215615

16. Karunaweera ND, Pratlong F, Siriwardane HV, Ihalamulla RL and Dedet JP. Sri Lankan cutaneous leishmaniasis is caused by Leishmania donovani zymodeme MON37. Trans $R$ Soc Trop Med Hyg 2003; 97(4):380-381. doi:10.1016/S00359203(03)90061-7

17. Siriwardana HVYD. Clinical epidemiology of leishmaniasis in Sri Lanka and the molecular identification of the causative organism. $\mathrm{PhD}$ thesis 2008.

18. Rajapaksa US, Ihalamulla RL and Karunaweera ND. First report of mucosal tissue localisation of leishmaniasis in Sri Lanka. Ceylon Med J 2005; 50(2):90-91. No doi

19. Abeygunesekara PH, Costa YJ, Seneviratne N, Ranatunga N and Wijesundera M de S. Locally acquired visceral leishmaniasis in Sri Lanka. Ceylon Med J 2007; 52(1):30-31. doi: $10.4038 /$ cmj.v52i1.1047

20. Rathnayake D, Ranawake RR, Sirimanna G, Siriwardhane Y, Karunaweera N and De Silva R. Co-infection of mucosal leishmaniasis and extra pulmonary tuberculosis in a patient with inherent immune deficiency. Int J Dermatol 2010; 49(5):549-51. doi:10.1111/j.1365-4632.2010.04376.x

21. Carter and Anthonypulle. Observations on sand flies in Delft island in North. Ceylon Med J. 1949; 43:62-73. doi:10.1111/j.1365-4632.2010.04376.x

22. Lane RP, Pile MM and Amerasinghe FP. Anthropophagy and aggregation behaviour of the sand fly phlebotomus argentipes in Sri Lanka. Med Vet Entomol 1990; 4:79-88. doi:10.1111/j.1365-2915.1990.tb00263.x

23. Lewis DJ and Killik Kendrik R. Some Phlebotomid sand flies of Malaysia and Sri Lanka. Trans $R$ Soc Trop Med Hyg 1973; 67(4):68-70. doi:10.1016/00359203(73)90258-7

24. Surendran SN, Karunaratne SH, Adams Z, Hemingway J and Hawkes NJ. Molecular and biochemical characterization of a sand fly population from Sri Lanka: evidence for insecticide resistance due to altered esterases and insensitive acetyl cholinesterase. Bull Entomol Res 2005; 95(4):371-80. doi:10.1079/BER2005368

25. Karunaweera ND, Abeysinghe R, Porter C and Dotson EM. Study on sandfly population in a cutaneous leishmaniasis focus in Sri Lanka. Proceedings of the $4^{\text {th }}$ World Congress on leishmaniasis, India 2009; 323.

26. Gajapathy K, Jude PJ and Surendran SN. Morphometric and meristic characterization of Phlebotomus argentipes species complex in northern Sri Lanka: evidence for the presence of potential leishmaniasis vectors in the country. Trop Biomed 2011; 28(2):259-268. No doi

27. Nawaratna SS, Weilgama DJ and Rajapaksha K . Cutaneous leishmaniasis in Sri Lanka: a study of possible animal reservoirs. Int J Infect Dis 2009; 13(4):513-517. doi:10.1016/j.ijid.2008.08.023 
28. Rosypal AC, Tripp S, Kinlaw C, Hailemariam S, Tidwell RR and Lindsay DS et al. Surveillance for antibodies to Leishmania spp. in dogs from Sri Lanka. J Parasitol. 2010; 96 (1):230-1. doi:10.1645/GE-2288

29. Siriwardana HVYD, Senanayake S, Weerasena KHW, Jayasinghe S and Karunaweera ND. Epidemiological study of leishmaniasis in a selected area in Sri Lanka. Proceedings of the $4^{\text {th }}$ World Congress on leishmanioses, India 2009; 96.

30. Perera WSR, Rajapakshe RPAS, Ihalamulla RL et al. Evaluation of Laboratory techniques used for the diagnosis of cutaneous leishmaniasis in Sri Lanka. Proceedings of the annual academic sessions of Sri Lanka College of Microbiologists 2004:31-32.

31. Ihalamulla RL, Siriwardana HVYD, Gamage S, Perera AJ and Karunaweera ND. First successful in- vitro cultivation of autochthonous Leishmania species in Sri Lanka. Ceylon Med J 2002; 47(2): 58. doi: http://dx.doi.org/10.4038/cmj.v47i2.3454

32. Ihalamulla RL, Siriwardana HV and Karunaweera ND. Efficacy of RPMI 1640 and M 199 media in the isolation of Leishmania from cutaneous lesions. Ann Trop Med Parasitol 2008; 102(2):173-175. doi:10.1179/136485908X252331

33. Siriwardana HVYD, Jayasinghe $S$ and Beeching, NJ, Chance ML and Karunaweera ND. A molecular tool for the diagnosis of cutaneous leishmaniasis in Sri Lanka. Proceedings of the annual academic sessions of Sri Lanka College of Microbiologists 2005:19-20. No doi

34. Harms G, Schonian G and Feldmeier H. Leishmaniasis in Germany. Emerg Infect Dis 2003; 9: 872-875. doi: 10.3201/eid0907.030023

35. Ben-Ami R, Schnur LF, Golan Y, Jaffe CL, Di T and Zeltser D. Cutaneous involvement in a rare case of adult visceral leishmaniasis acquired in Israel. $J$ Infect 2002; 44(3):181-184. doi:10.1053/jinf.2002.0953

36. Sethuraman G, Sharma VK and Salotra P. Indian mucosal leishmaniasis due to Leishmania donovani infection. New Eng Journal of Med 2008; 358(3):313-315. doi:10.1056/NEJMc072391

37. Pearson RD and De Queiroz Sousa A. Leishmania species: visceral (kala-azar), cutaneous and mucosal leishmaniasis. In: Mandell GL, Bennett JE and Dolin R (eds). Principles and Practice of Infectious Diseases. (4th ed). New York: Churchill Livingstone. 1995:2434.

38. Guevara P, Ramirez JL, Rojas L, Scorza JV, Gonzales N and Anez N. Leishmania braziliensis in blood 30 years after cure. Lancet 1993; 341:1341. doi:10.1016/01406736(93)90845-8

39. Dedet JP and Pratlong F. Leishmaniasis. In: Gordon Cook and Alimuddin Zumla. (Eds). Manson's Tropical Diseases (21 ${ }^{\text {st }}$ ed). Saunders 2002; 1339-1364.

40. Mebrahtu YB, Van Eys G and Guizani I et al. Human cutaneous leishmaniasis caused by Leishmania donovani s.l. in Kenya. Trans R Soc Trop Med Hyg 1993; 87(5):598601. doi:10.1016/0035-9203(93)90101-U

41. Castellani A. Leishmania donovani in Ceylon Med J 1904; 37:217-343.

42. Herwaldt H. Leishmaniasis. Lancet 1999; 354:1191-1199. doi:10.1016/S01406736(98)10178-2

43. Chappuis F, Sundar S, Hailu A, Ghalib H, Rijal S and Peeling RW et al. Visceral leishmaniasis: what are the needs for diagnosis, treatment and control? Nat Rev Microbiol 2007; 5: 873- 882. doi:10.1038/nrmicro1748

44. Report of World Health Organization on the workshop on control of visceral leishmaniasis, WHO/Leish/96:40. 1996. World Health Organization, Geneva, Switzerland. 
45. Loo WJ, Chan SK, Rytina E, Lockwood DNJ, Sterling JC and Todd P. Five cases of cutaneous leishmaniasis in Cambridge, UK. Br J Dermatol 2005; 153:1067-1092. doi:10.1111/j.1365-2133.2005.06921.x

46. Report of the scientific working group on leishmaniasis. World Health Organization. Geneva. Switzerland. 2004.www.who.int/tdr

47. Sundar S, Singh VP, Sharma S, Makharia MK and Murray HW. Response to interferon-gamma plus pentavalent antimony in Indian visceral leishmaniasis. $J$ Infect Dis 1997; 176(4):1117-1119. doi:10.1086/516526

48. Sadeghian G, Iraji F and Anilfroushzadeh I. Disseminated cutaneous leishmaniasis on lymphedema following radiotherapy. Int $J$ Dermatol 2005; 44:610-611. doi:10.1111/j.1365-4632.2004.02350.x

49. González U, Pinart M, Reveiz L and Alvar J and Tweed JA. Interventions for Old World cutaneous leishmaniasis. Cochrane Database Syst Rev 2008 Issue 4 doi:10.1002/14651858.CD005067.pub3

50. Croft $\mathrm{S}$ and Coombs GH. Leishmaniasis-current chemotherapy and recent advances in the search for novel drugs. Trends Parasitol 2003; 19(11):502-508. doi:10.1016/j.pt.2003.09.008

51. Bryceson A. A policy for leishmaniasis with respect to prevention and control of drug resistance. Trop Med Int Health 2001; 6: 928-934. doi:10.1046/j.1365-3156.2001.00795.x

52. Thakur CP, National level policy issues: In: Thakur H (ed). Kala Azar - Emerging Perspectives and Prospects in South Asia. Centre for Health Policy, Planning and Management, School of Health Systems Studies, Tata Institute of Social Sciences (TISS) (2011); 311-369.

53. Sanyal RK. Some observations on epidemiology of current outbreak of kala-azar in Bihar. J Comm Dis. 1979; 11: 170-82. No doi

54. Das M, Banjara M, Chowdhury R, Ku V, Rijal S and Joshi A. Visceral leishmaniasis on the Indian sub-continent: a multi-centre study of the costs of three interventions for the control of the sand fly vector, Phlebotomus argentipes. Ann Trop Med Parasitol 2008; 102 (8):729-41. doi:10.1179/136485908X355274

55. Ranasinghe PHKIS, Abeygunasekera PH, Athauda SB, Chandrasekharan NV, Mendis ASV, Hulangamuwa CS, Wickremasinghe DR. First successful in vitro culture of Leishmaniasp. causing autochthonous visceral leishmaniasis in Sri Lanka Cey. Med. J. 2011; 56 (4): 179-180. doi: http://dx.doi.org/10.4038/cmj.v56i4.3904 